\title{
Differential Expression of HSP90 $\beta$ in MDA-MB-231 and MCF-7 Cell Lines after Treatment with Doxorubicin
}

\author{
Fereshte Jokar' ${ }^{1}$, Javad Amini Mahabadi², Morteza Salimian³, Aliakbar Taherian', \\ Seyyed Mohammad Gheibi Hayat ${ }^{4}$, Amirhossein Sahebkar ${ }^{5,6,7}$, Mohammad Ali Atlasi' * \\ ${ }^{1}$ Anatomical Sciences Research Center, Kashan University of Medical sciences, Kashan, Iran \\ ${ }^{2}$ Gametogenesis Research Center, Kashan University of Medical Sciences, Kashan, Iran \\ ${ }^{3}$ Platelet Research Laboratory, Kashan University of Medical Science, Kashan, Iran \\ ${ }^{4}$ Department of Medical Genetics, School of Medicine, Shahid Sadoughi University of Medical Sciences, Yazd, Iran \\ ${ }^{5}$ Neurogenic Inflammation Research Center, Mashhad University of Medical Sciences, Mashhad, Iran \\ ${ }^{6}$ Biotechnology Research Center, Pharmaceutical Technology Institute, Mashhad University of Medical Sciences, Mashhad, Iran \\ ${ }^{7}$ School of Pharmacy, Mashhad University of Medical Sciences, Mashhad, Iran
}

\section{Key Words}

HSP90a, HSP90 $\beta$, MCF-7, MDA-MB-231, breast cancer, heat shock protein

\begin{abstract}
Background: Breast cancer is a complex, heterogeneous disease and one of the most common malignancies in women worldwide. The efficacy of chemotherapy as an important breast cancer treatment option has been severely limited because of the inherent or acquired resistance of cancer cells. The molecular chaperone heat shock protein 90 (HSP90) upregulated in response to cellular stress is required for functions such as conformational maturation, activation and stability in more than 200 client proteins, mostly of the signaling type. In this study, the expression of HSP90 isoforms including HSP90 $\alpha$ and HSP90 $\beta$ in breast cancer cell lines before and after treatment with doxorubicin (DOX) was assessed.
\end{abstract}

Material and Methods: The cell cytotoxicity of DOX in

Received: Apr 21, 2018 Reviewed: Oct 19, 2018 Accepted: Feb 11, 2019

(c) This is an Open-Access article distributed under the terms of the Creative Common Attribution Non-Commercial License (http://creativecommons.org/licenses/by-nc/4.0/) which permits unrestricted noncommercial use, distribution, and reproduction in any medium, provided the original work is properly cited.

( $)$ This paper meets the requirements of KS X ISO 9706, ISO 9706-1994 and ANSI/NISO Z39.48-1992 (Permanence of Paper).
MDA-MB-231 and MCF-7 cell lines was determined using the MTT assay. Immunofluorescence and western blotting techniques were used to determine the expression of HSP90 $\beta$ in the cell lines before and after DOX treatment. Immunofluorescence was also conducted to ascertain the expression of HSP90a.

Results: The MTT assay results showed that the MDAMB-231 cells $\left(\mathrm{IC}_{50}=14.521 \mu \mathrm{M}\right)$ were more sensitive than the MCF-7 cells $\left(\mathrm{IC}_{50}=16.3315 \mu \mathrm{M}\right)$ to DOX. The immunofluorescence results indicated that the expression of HSP90a in both cell lines decreased after exposure to DOX. The western blot and immunofluorescence analyses showed that HSP90 $\beta$ expression decreased in the MCF-7 cells but increased in the MDAMB-231 cells after DOX treatment. Conclusion: The obtained results suggested that HSP90a and HSP90 $\beta$ expression levels were reduced in the MCF-7 cells after exposure to DOX. In the MDA-MB-231 cells, HSP90a expression was reduced while HSP90 $\beta$ was found to be overexpressed following DOX treatment.

\section{Introduction}

Breast cancer is a complex and heterogeneous disease and one of the most common malignancies in 
women worldwide. A total of 234,190 new breast cancer cases and 40,730 cancer deaths were anticipated to occur in the United States in 2015 [1, 2]. Such a high prevalence reflects the limited efficacy of chemotherapy, which can be primarily attributed to the development of resistance to chemotherapeutic drugs. Chemotherapeutic drugs such as doxorubicin (DOX) are generally used to treat a variety of malignancies such as breast cancer but the drug resistance acquired by cancer cells can lead to treatment failure as surviving cancer cells become refractory to treatment and contribute to recurring and increased metastatic activity [3-6]. There is currently an inadequate understanding of the molecular and cellular mechanisms that govern breast cancer and the mechanisms underlying the chemotherapy resistance.

The heat shock proteins assist other proteins under normal and stress conditions to manage several actions including correct folding, assembly, intracellular localization, secretion, regulation and degradation [7]. Among these proteins, the molecular chaperone HSP90 with more than 200 target proteins has gained interest as a promising anti-cancer drug target, as many of these target proteins play an important role in disrupted pathways involved in different types of cancer [8]. Two highly elevated vertebrate HSP90 isoforms, namely HSP90 $\alpha$ and HSP90 $\beta$, are encoded by human genes $[9,10]$.

The breast cancer cell lines can be representative of the key genetic and transcriptional features involved in the breast tumors [11-14]. The pathobiology, screening and therapeutic features of the breast cancer can be examined using these cell lines $[15,16]$, which have several advantages such as the relative ease of culturing, the possibility of being pharmacologically functional assays, and purity of cancerous cells or non-contaminated stromal cell [14].

The present study aimed to investigate two different breast cancer cell lines, MCF-7 and MDA-MB-231. MCF7 cells are estrogen receptor (ER)-positive cells that have been widely used to study estrogen-related pathways and the development of anti-hormonal therapies for breast cancer [17-19]. The MDA-MB231 cell line is ER-negative and resembles the recently characterized claudin-low tumor subtype of breast cancer [20,21]. Additionally, the MCF-7 originates from metastatic sites in adenocarcinoma, as a tumorigenic adherent breast epithelial cell line, whereas the MDA-MB-231 cell line is a tumorigenic metastatic breast cell line [22].

Studies are continuously conducted worldwide to identify the critical factors involved in cancer resistance to chemotherapy. To supplement existing efforts, the current study was carried out to compare the expression of HSP90 $\alpha$ and HSP90 $\beta$ in MDA-MB-231 and MCF-7 cells after treatment with DOX.

\section{Materials and Methods}

Antibodies against HSP90 $\beta$ (sc-1057) and HSP90a (sc8262) and HRP secondary antibodies (sc-2354) and FITC-conjugated secondary antibodies (sc-2988) were obtained from Santa Cruz Biotechnology (Santa Cruz Biotechnology, Inc., Texas and Santa Cruz Biotechnology, Inc., California, respectively). A beta actin antibody (ab8502) was obtained from United States Abcam. DOX (D1515) and MTT powder (M2128) were purchased from Germany Sigma.

\subsection{Breast cancer cell lines}

The MDA-MB-231 and MCF-7 cell lines (prepared from Pasture Institute of Iran) were initially cultured in RPMI 1640 medium in the presence of $10 \%$ FBS and $100 \mathrm{U} / \mathrm{ml}$ of penicillin/streptomycin. The incubation was performed at $37^{\circ} \mathrm{C}$ with $5 \% \mathrm{CO}_{2}$ and humidity.

\subsection{MTT assay}

A colorimetric assay with tetrazolium salt was used to assess the anti-proliferative effects of DOX. The breast cancer cells were seeded at 10,000 cells/well in 96-well plates and allowed to grow for $48 \mathrm{~h}$. The cells were then treated with different concentrations of DOX for $24 \mathrm{~h}$, after which each well was poured by $10 \mu \mathrm{l}$ of MTT [3-(4, 5-dimethylthiazol-2-yl)-2, 5- diphenyltetrazolium bromide] and 5 $\mathrm{mg} / \mathrm{ml}$ in PBS (phosphate buffered saline) solution, and incubation was done at $37^{\circ} \mathrm{C}$ for 4 hours. After dissolving the formazan crystals in the DMSO, a microplate reader was used to read the absorbance of the wells at $570 \mathrm{~nm}$. Finally, the data were reported as means \pm standard deviation for all experiments with more than three replicates, and statistically analyzed using SPSS software. The linear regression analysis was applied to estimate the half-maximal inhibitory concentrations $\left(\mathrm{IC}_{50}\right)$ from the in vitro dose-response curves.

\subsection{Western blotting}

The MCF-7 and MDA-MB-231 cells were cultured in 100 $\mathrm{mm}$ plates and treated with $\operatorname{DOX}(0,0.2,5$ and $10 \mu \mathrm{M})$ for $24 \mathrm{~h}$. The cells were rinsed with cold PBS once and lysed with $500 \mu \mathrm{l}$ of RIPA buffer ( $50 \mathrm{mM}$ of Tris, $\mathrm{pH}=8,150 \mathrm{mM}$ of $\mathrm{NaCl}, 0.1 \%$ SDS, $0.5 \% \mathrm{Na}$ deoxycholic acid, $1 \% \mathrm{NP}-40$ or IGEPAL, $10 \mathrm{~g} / \mathrm{ml}$ of aprotinin and $10 \mathrm{~g} / \mathrm{ml}$ of leupeptin). A 25 G 5/8 needle was employed to break the cells whose extract was centrifuged for $30 \mathrm{~min}$ at 14,000 rpm at $4^{\circ} \mathrm{C}$. Then, the resulting supernatant was stored at $-20^{\circ} \mathrm{C}$ until testing. Bradford method was followed to obtain the protein concentration. In addition, $24 \mu \mathrm{g}$ proteins per well were electrophoresed using SDS polyacrylamide gels, and were then delivered onto a nitrocellulose membrane with a semi-dry gel transfer apparatus. Next, 5\% milk in PBST (PBS with $0.05 \%$ Tween 20) was used to block the membranes at room temperature for 1-2 hours, and subsequently was incubated in the presence of a primary antibody against $\mathrm{HSP} 90 \beta$ or $\beta$-actin at $4^{\circ} \mathrm{C}$ overnight. After that, the membranes were washed with PBST for 10 min. The incubation was performed for an hour with the HRP-labeled secondary antibody in 5\% milk in PBST. The washing was continued with PBST for 20-30 min, and then the membranes were treated with chemiluminescence reagents and exposed to Kodak film. 


\subsection{Immunofluorescence}

The study cell lines after culturing on coverslips within cell culture plates were treated at $0,0.2,5,10 \mu \mathrm{M}$ concentrations of DOX and incubated for 24 hours. In the next step, the cells following washing by PBS were fixed with 4\% paraformaldehyde/PBS at ambient temperature for 15 $\mathrm{min}$. The cells were rewashed and exposed to immunofluorescence staining with a polyclonal primary antibody against HSP90 $\beta$ and HSP90 $\beta$ followed by the corresponding FITC-conjugated secondary antibody. The stained cells were examined using a fluorescence microscope.

\subsection{Statistical analysis}

The data obtained from the MTT assay were plotted. Also, linear charts were drawn; and the average, standard deviation, standard error, and $\mathrm{IC}_{50}$ values were calculated. Total Lab software was used for the densitometry of the bands. All values were presented as mean \pm SEM from three independent experiments, and statistically significant differences were determined among various groups by ANOVA and Tukey posttest using SPSS 12.0 statistical software.

\section{Results}

The cytotoxic effects of DOX on the proliferation of the MCF-7 and MDA-MB-231 cell lines were evaluated by MTT assay. The $\mathrm{IC}_{50}$ of each cell line was calculated via linear regression. The results showed that the MDA-MB-231 cells $\left(\mathrm{IC}_{50}=14.521 \mu \mathrm{M}\right)$ were more sensitive than the MCF-7 cells $\left(\mathrm{IC}_{50}=16.3315 \mu \mathrm{M}\right)$ to $24 \mathrm{~h}$ DOX treatment.

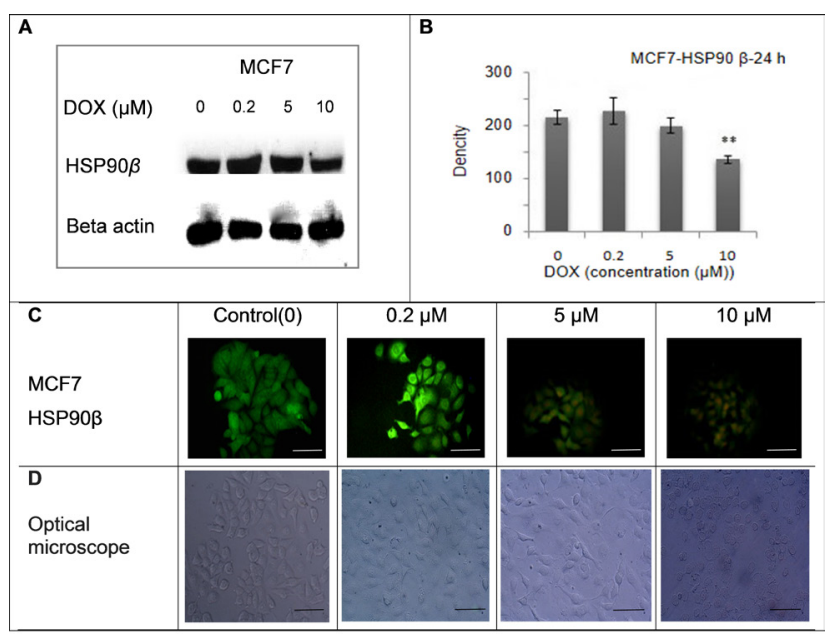

Figure 1 These data show the expression of HSP90 $\beta$ before and after treatment with different concentrations of $\operatorname{DOX}(0,0.2,5,10$ $\mu \mathrm{M})$ in MCF7 cell line for $24 \mathrm{~h}$. The expression of HSP90 $\beta$ in MCF7 cells was increased in concentration of $0.2 \mu \mathrm{M}$ and decreased in concentration of 5 and $10 \mu \mathrm{M}$ and shows MCF7 cell density by optical microscope (x 100). The scale bar represents $100 \mu \mathrm{m}$.

The expression of HSP90 $\beta$ before and after treatment with different concentrations of DOX was assayed using western blot analysis. The data from the western blot analysis showed that HSP90 $\beta$ expression decreased in

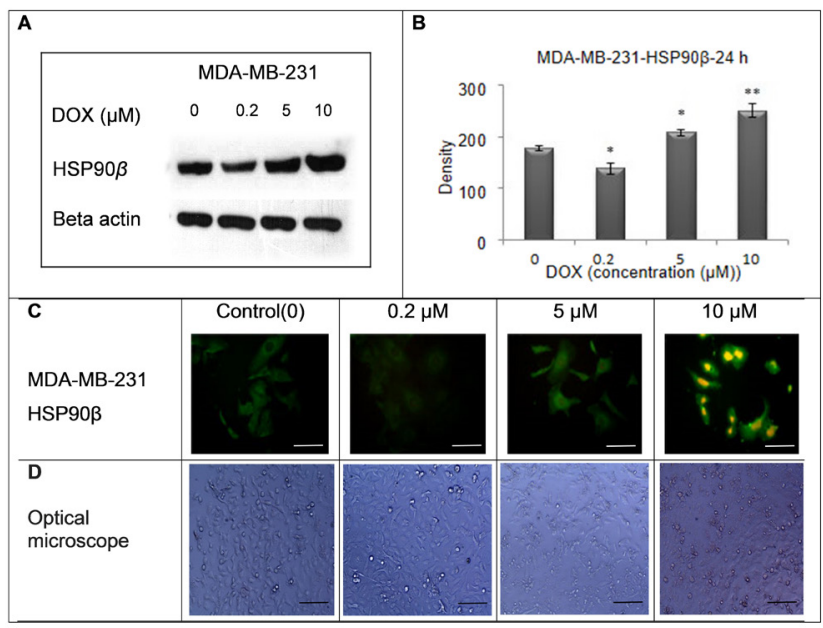

Figure 2 These data show the expression of HSP90 $\beta$ before and after treatment with different concentrations of DOX $(0,0.2,5$, and $10 \mu \mathrm{M}$ ) in MDA-MB-231 cell line for $24 \mathrm{~h}$. The expression of HSP90 $\beta$ in MDA-MB-231 cells was decreased in the concentration of $0.2 \mu \mathrm{M}$ and increased in the concentrations of 5 and $10 \mu \mathrm{M}$ and shows MDA-MB-231 cell density by optical microscope (x 100). The scale bar represents $100 \mu \mathrm{m}$.

the MCF-7 cells ( 5 and $10 \mu \mathrm{M}$ DOX) but increased in the MDA-MB-231 cells (5 and $10 \mu \mathrm{M}$ DOX) (Figures 1 and 2). The MCF-7 curve showed that HSP90 $\beta$ expression significantly decreased at a DOX concentration of $10 \mu \mathrm{M}(\mathrm{P}<$ 0.01 ) (Figure 1-B). The MDA-MD-231 curve indicated that HSP90 $\beta$ expression significantly increased at DOX concentrations of $5 \mu \mathrm{M}\left({ }^{*} \mathrm{P}<0.05\right)$ and $10 \mu \mathrm{M}(\mathrm{P}<0.01)$ (Figure $2-\mathrm{B})$. The western blotting method was used to detect the HSP90 $\beta$ expression in the MCF-7 cells. Figure 2 (A) shows a sample of blots regarding three independent tests. The data, expressed in means \pm standard deviation $(n=3)$, indicated that HSP90 $\beta$ expression significantly declined $(\mathrm{P}<0.01)$ at a DOX concentration of $10 \mu \mathrm{M}$, as shown in (B). The representative immunofluorescence images (100X magnification) of the MCF-7 cells are presented in (C). Based on the the optical microscope, cell density decreased after exposure to DOX. Cytoplasmic ventricles and cellular attachments decreased was observed in the cells (D).

The western blotting was applied to detect the HSP90 $\beta$ expression in the MDA-MB-231 cells. Figure 2 (A) illustrates a sample of blots regarding three independent tests. The data, expressed as means \pm standard deviation $(n=3)$, revealed that the upper expression of HSP90 $\beta$ was significant at DOX concentrations of $5 \mu \mathrm{M}(\mathrm{P}<0.05)$ and $10 \mu \mathrm{M}$ $(\mathrm{P}<0.01)$, as shown in (B). The representative immunofluorescence images (100X magnification) of the MDAMB-231 cells are provided in (C). Based on the images seen from the optical microscope, cell density decreased after DOX exposure. Cytoplasmic ventricles and cellular attachments decreased as dosage increased, and cell death was observed in the cells (D).

The immunofluorescence and western blot results showed that the MDA-MB-231 and MCF- 7 cells had different levels of HSP90 $\beta$ expression in response to DOX treatment. In the MCF-7 cells, HSP90 $\beta$ expression decreased 
after DOX treatment. Although expression increased at a DOX concentration of $0.2 \mu \mathrm{M}$, the $10 \mu \mathrm{M}$ dose of DOX significantly reduced such expression. In the MDA-MB-231 cells, HSP90a expression increased after DOX treatment. Expression decreased at a DOX concentration of $0.2 \mu \mathrm{M}$ but significantly increased at DOX doses of 5 and $10 \mu \mathrm{M}$ (Figures 1 and 2). The expression of HSP90 $\beta$ before and after exposure to DOX was determined via immunofluorescence. The findings revealed that HSP90 $\alpha$ was expressed in the nucleus and cytoplasm before exposure to DOX but that expression decreased after both cell lines were exposed to DOX. In the MDA-MB-231 cells, the expression of HSP90 $\alpha$ decreased even further (Figure 3).

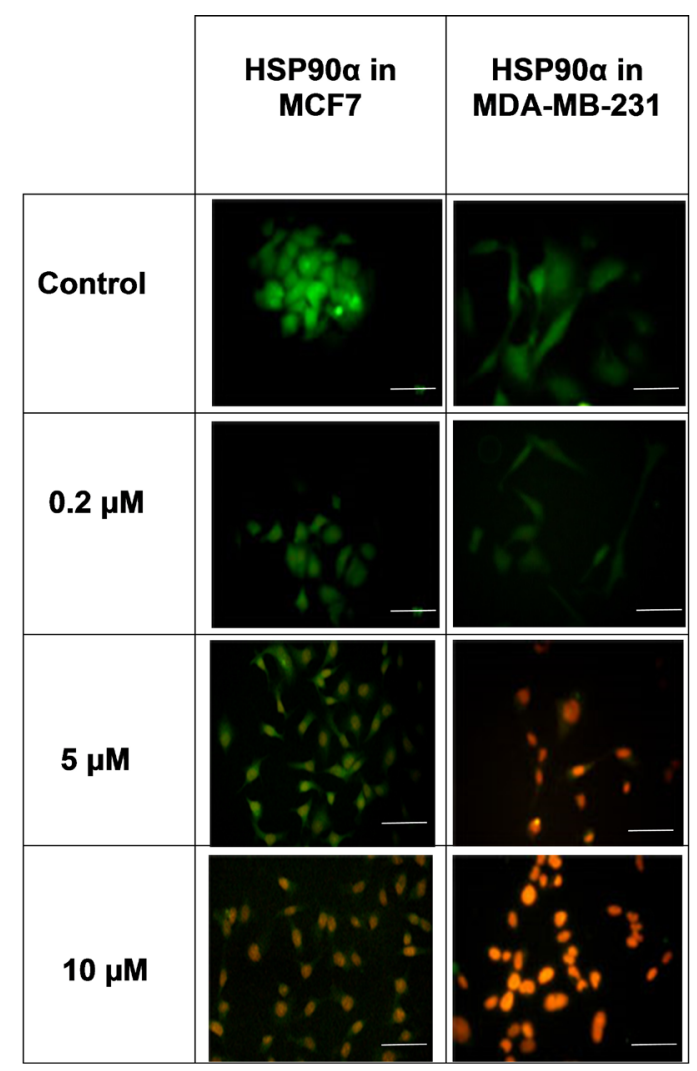

Figure 3 The expression of HSP90a before and after exposure to Dox was measured. The expression of HSP90a was assessed before exposure to doxorubicin in the nucleus and cytoplasm but decreased after exposure to Dox in both cell lines. The scale bar represents $100 \mu \mathrm{m}$.

\section{Discussion}

Breast cancer is a heterogeneous disease with several subtypes, whose current classification may be incomplete or unreliable given that patients with apparently identical types of breast cancer respond differently to the same treatment [2]. Chemotherapy resistance remains an unsolved clinical problem in cancer treatment. Studying different pathways involved in chemotherapy may enable an improved understanding of the mechanism that govern breast cancer and may shed light on more reliable biomarkers. In this research, the expression of HSP90 $\beta$ in
MDA-MB-231 and MCF-7 cells before and after treatment with different concentrations of DOX was assayed. In this study, HSP90a protein expression was measured by the immunofluorescence technique in both cell lines before and after exposure to DOX. The toxicity of DOX against the two cell lines was compared using the MTT assay. The comparison of the IC50 values showed that the MDAMB-231 cells $(14.521 \mu \mathrm{M})$ were more sensitive than the MCF-7 cells ( $16.3315 \mu \mathrm{M})$ to the treatment.

The expression of HSP90 $\alpha$ in both cell lines decreased after exposure to DOX. In the MCF-7 cells, DOX decreased the expression of HSP90 $\beta$ in a dose-dependent manner but in the MDA- MB-231 cells, HSP90 $\beta$ expression increased after chemotherapy. In the MDA-MB-231 cells, HSP90 $\beta$ expression decreased with decreasing DOX dose but higher doses induced higher expression. Georgakis et al. tested the effect of an HSP90 inhibitor in anaplastic large cell lymphoma (ALCL) cells. Besides, they inhibited HSP90 without discerning the seemingly different roles of HSP90 isoforms [23]. However, the results of the cited study cannot be directly compared with the present results unless a specific HSP90 isoform inhibitor is used in breast cancer cell lines.

Interestingly, different doses of a chemical sometimes induce opposite responses. For example, in immunofluorescence and western blotting images of MDA-MB-231 cells after exposure to $0.2 \mu \mathrm{M}$ doxorubicin, the expression of HSP90 $\beta$ initially decreases but its expression increases with increasing drug concentrations (Figure 2). Also, in MCF-7 cells after exposure to the concentration of $0.2 \mu \mathrm{M}$ doxorubicin, the expression of HSP90 $\beta$ increased but its expression decreased as the concentration of the drug increased (Figure 3). As observed in the MDA-MB-231 cells, a lower dose of DOX reduced HSP90 $\beta$ expression but a higher dose increased the expression. The most important insight derived from our results was that after chemotherapy, HSP90 $\beta$ expression decreased in the MCF-7 cells but increased in the MDA- MB-231 cells. The reduced expression of HSP90 $\beta$ was expected in general after chemotherapy but its increased expression in the MDA-MB-231 cells is interesting and should be investigated in more details in future studies. The HSP90 serves as a favorable therapeutic target in studying a wide range of cancers. In the early stages of oncogenesis and the maintenance stage of malignant cell phenotypes, high HSP90 expression can be seen [24]. Hence, induction of apoptosis has been shown to be one of the leading approaches in anti-tumor therapy $[25,26]$.

In a research, the therapeutic potential of sulforaphane (SFN), an antioxidant derived from broccoli, was evaluated against a chemoresistant cell line (BEAC) in vivo [27]. Immunodeficient mice were injected with BEAC cells, and after the appearance of palpable tumors, the mice were treated with SFN. The SFN induced cell apoptosis, suppressed the expression of multidrug resistance proteins, and reduced tumor volume. The anticancer activity of SFN can induce caspase 8 and p21 and downregulates the HSP90 [28].

In another research, the differential expression of HSP90a and HSP90 $\beta$ in other cancer cells were recorded. A northern blot analysis showed that the levels of HSP90 $\beta$ and HSP90a mRNA in HL60 cells that are resistant to methotrexate or cisplatin were approximately two-to three-fold 
higher than those in a drug-sensitive parental cell line [29]. In another work, the DOX- resistant colon cancer cell line LoVoDx ${ }^{R}$ expressed a high level of HSP90 $\beta$ constitutively but in a sensitive cell line, no constitutive expression of HSP90 $\beta$ was found [30]. An immunohistochemical study on colorectal cancer patients ( 26 men and 23 women) before chemotherapy showed the expression of HSP90a and HSP60 in the cytoplasm and the expression of HSP70 in the nucleus of paracancerous tissue; the research also identified the expression of HSP60 and HSP70 in the cytoplasm and the expression of HSP90a in the nucleolus and extracellular matrix of cancerous tissue [31]. In another research, the mRNA expression of HSP60, HSP90a and HSP70 between precancerous and cancerous tissues was compared using RT-PCR. The results showed that HSP60, HSP70 and HSP90a mRNA levels were more highly expressed in cancerous tissue than in precancerous tissue. Western blot analysis confirmed the higher level of these three proteins in cancerous tissue [32, 33]. In another study, HSP90a protein was more highly expressed in cancerous tissue than in non- cancerous tissue [34]. Song et al. (2010) reported that the expression of HSP90 and PI3Ka-P110a was higher in the patients with ER/PR-positive and Her-2-negative breast cancer compared to the patients with ER/PR-negative or Her-2-positive breast cancer. The authors suggested HSP90 and PI3Ka-p110a as molecular prognostic markers for predicting early relapse in patients suffering from breast cancer [35].

Cell line-specific factors may be part of the reason for the differences in results between the MDA-MB-231 and MCF-7 cells in the current study. MCF-7 cells express estrogen and progesterone receptors whereas MDA-MB-231 cells do not express any of these receptors [19]. One of the major subtypes of breast cancer is that which expresses steroid hormone receptors [36]. Steroid hormone receptors were found to be expressed in $65 \%$ and $80 \%$ of breast cancer patients before and after menopause, respectively. This means that steroid hormone receptors are expressed, on average, in $75 \%$ of breast cancer patients [37]. In another study, the expression of Erk and pErk in MDAMB-231 and MCF-7 cells was compared after chemotherapy with DOX or docetaxel. The results showed that the MDA-MB-231 and MCF- 7 cells responded in completely different ways to the same treatment. The expression of Erk and pErk in the MDA-MB-231 cells expression in the MCF-7 cells increased in a dose-dependent manner after chemotherapy. The expression of pErk decreased in the MDA-MB-231 cells but increased in the MCF-7 cells after docetaxel treatment [38].

The HSP90 isoform-specific interaction with client proteins has also been reported in different studies. Co-immunoprecipitation studies in two different systems showed that both isoforms interacted similarly with different co-chaperones in the HSP90 heterocomplex. Some of the substrates (CKIIB, c-Src, A-Raf and Erk) interacted similarly with both isoforms but after heat shock, they exhibited stronger interaction with HSP90 $\alpha$ [39]. One of the HSP90 $\beta$ client proteins is the cellular inhibitor of apoptosis protein-1 (c-IAP1). Inhibition of HSP90 proteins by small chemical molecules and the specific depletion of HSP90 $\beta$ by siRNA results in c-IAP1 auto- ubiquitination and degradation by the protea- some machinery.

The $\beta$ isoform accounts for the chaperone function of HSP90 toward c-IAP1 since the specific HSP90 $\alpha$ depletion had no effect on the c-IAP1 percentage [40]. In a study by Dong et al. who investigated MDA-MB-231 breast cancer cell hypoxia in vivo, the inhibition of HSP90a by monoclonal antibody 1G6-D7 increased tumor cell death. They found that the expression of HSP90a secretion is an influential factor in the survival of hypoxic tumor cells [41]. In a study in the presence of B lymphocyte in inflammatory infiltration, estrogen and progesterone receptors disappeared, resulting in the accumulation of HSP90 in the cell and contributing to the stabilization of HER2/neu receptors and the most prominent proteins that led to tumor progression [42].

Overall, the studies discussed above have shown that HSP90 expression is important and the expression of HSP90 $\beta$ proceeds in a different manner in chemotherapy-resistant cells.

\section{Conclusion}

In this study, we observed decreased cellular density in both tested cell types after exposure to DOX. The expression of HSP90a in both cell lines was also decreased after exposure to DOX. The HSP90 $\beta$ expression was reduced in the MCF-7 cells but increased in the MDA-MB- 231 cells after DOX treatment. These findings may be attributed to the greater sensitivity of the MDA-MB-231 cells to DOX than the MCF-7 cells or the absence of ERs in MDAMB-231 cells. Overall, the present results serve as preliminary data for conducting future proof-of- concept studies to explore the role of HSP90 isoforms and estrogen receptors in the sensitivity of breast cancer cells, cancer cell isolates and xenografts to chemotherapeutic agents. Future studies are warranted to test the effect of overexpression of HSP90 $\beta$ on DOX sensitivity in MCF-7 cells, or the effect of knockout of HSP90 $\beta$ on the sensitivity of MDA-MB-231 cells to DOX. Finally, it remains to be explored if HSP90 $\beta$ affects intracellular DOX accumulation in MDA-MB-231 cells and through this potential mechanism regulates the sensitivity of cells to this drug.

\section{Acknowledgments}

The Research Deputy of Kashan University of Medical Sciences and Kashan Anatomical Sciences Research Center funded the present research project with the grant code 9294. The authors would like to thank the personnel of the medical laboratory at Kashan University of Medical Sciences and the Anatomical Sciences Research Center for their collaboration in the current study.

\section{Conflict of interest}

The authors declare no conflict of interest. 


\section{References}

1. Siegel RL, Miller KD, Jemal A. Cancer statistics, 2015. CA: a cancer journal for clinicians. 2015;65(1):5-29.

2. Veronesi U, et al. Rethinking TNM: a breast cancer classification to guide to treatment and facilitate research. The breast journal. 2009;15(3):291-295.

3. Longley D, Johnston P. Molecular mechanisms of drug resistance. The Journal of pathology. 2005;205(2):275-292.

4. Aas T, et al. Specific P53 mutations are associated with de novo resistance to doxorubicin in breast cancer patients. Nature medicine. 1996;2(7):811-814.

5. Harris AL, Hochhauser D. Mechanisms of multidrug resistance in cancer treatment. Acta Oncologica. 1992;31(2):205-213.

6. Jacks T, Weinberg RA. Taking the study of cancer cell survival to a new dimension. Cell. 2002;111(7):923-925.

7. Joly AL, et al. Dual role of heat shock proteins as regulators of apoptosis and innate immunity. Journal of Innate Immunity. 2009;2(3):238-247.

8. Echeverria PC, et al. Detection of changes in gene regulatory patterns, elicited by perturbations of the Hsp90 molecular chaperone complex, by visualizing multiple experiments with an animation. BioData Min. 2011;4(1):15.

9. Hickey E, et al. Sequence and regulation of a gene encoding a human 89-kilodalton heat shock protein. Mol Cell Biol. 1989;9(6):2615-26.

10. Rebbe NF, et al. Nucleotide sequence and regulation of a human $90-\mathrm{kDa}$ heat shock protein gene. Journal of $\mathrm{Bi}$ ological Chemistry. 1989;264(25):15006-15011.

11. Neve RM, et al. A collection of breast cancer cell lines for the study of functionally distinct cancer subtypes. Cancer cell. 2006;10(6):515-527.

12. Chin K, et al. Genomic and transcriptional aberrations linked to breast cancer pathophysiologies. Cancer cell. 2006;10(6):529-541.

13. Hollestelle A, et al. Distinct gene mutation profiles among luminal-type and basal-type breast cancer cell lines. Breast cancer research and treatment. 2010;121(1):53-64.

14. Kao J, et al. Molecular profiling of breast cancer cell lines defines relevant tumor models and provides a resource for cancer gene discovery. PloS one. 2009;4(7):e6146.

15. Lacroix M, Leclercq G. Relevance of breast cancer cell lines as models for breast tumours: an update. Breast cancer research and treatment. 2004;83(3):249-289.

16. Vargo-Gogola T, Rosen JM. Modelling breast cancer: one size does not fit all. Nature Reviews Cancer. 2007;7(9):659-672.

17. Soule $\mathrm{H}$, et al. A human cell line from a pleural effusion derived from a breast carcinoma. Journal of the National Cancer Institute. 1973;51(5):1409-1416.

18. Wood AJ, Osborne CK. Tamoxifen in the treatment of breast cancer. New England Journal of Medicine. 1998;339(22):1609-1618.

19. Fabbro D, et al. Epidermal growth factor binding and protein kinase $\mathrm{C}$ activities in human breast cancer cell lines: possible quantitative relationship. Cancer research. 1986;46(6):2720-2725.
20. Prat A, et al. Phenotypic and molecular characterization of the claudin-low intrinsic subtype of breast cancer. Breast Cancer Res. 2010;12(5):R68.

21. Heiser LM, et al. Subtype and pathway specific responses to anticancer compounds in breast cancer. Proceedings of the National Academy of Sciences. 2012;109(8):2724-2729.

22. Visagie M, Mqoco T, Joubert A. Sulphamoylated estradiol analogue induces antiproliferative activity and apoptosis in breast cell lines. Cellular and Molecular Biology Letters. 2012;17(4):549-558.

23. Georgakis GV, et al. The HSP90 inhibitor 17-AAG synergizes with doxorubicin and U0126 in anaplastic large cell lymphoma irrespective of ALK expression. Exp Hematol. 2006;34(12):1670-9.

24. Sõti $\mathrm{C}$, et al. Heat shock proteins as emerging therapeutic targets. British journal of pharmacology. 2005;146(6):769-780.

25. Ghobrial IM, Witzig TE, Adjei AA. Targeting apoptosis pathways in cancer therapy. CA: a cancer journal for clinicians. 2005;55(3):178-194.

26. Guo W, et al. Targeting GRP75 improves HSP90 inhibitor efficacy by enhancing p53-mediated apoptosis in hepatocellular carcinoma. PloS one. 2014;9(1):e85766.

27. Ullah MF. Sulforaphane (SFN): an isothiocyanate in a cancer chemoprevention paradigm. Medicines. 2015;2(3):141-156.

28. Qazi A, et al. Anticancer activity of a broccoli derivative, sulforaphane, in barrett adenocarcinoma: potential use in chemoprevention and as adjuvant in chemotherapy. Transl Oncol. 2010;3(6):389-99.

29. Beyer-Sehlmeyer G, et al. Suppressive subtractive hybridisation reveals differential expression of serglycin, sorcin, bone marrow proteoglycan and prostate-tumour-inducing gene I (PTI-1) in drug-resistant and sensitive tumour cell lines of haematopoetic origin. Eur J Cancer. 1999;35(12):1735-42.

30. Bertram J, et al. Overexpression of ribosomal proteins L4 and L5 and the putative alternative elongation factor PTI-1 in the doxorubicin resistant human colon cancer cell line LoVoDxR. Eur J Cancer. 1998;34(5):731-6.

31. Zhang W, et al. Expressions of heat shock protein (HSP) family HSP 60, 70 and 90 a in colorectal cancer tissues and their correlations to pathohistological characteristics. Chinese J Cancer. 2009;28:1-7.

32. Lim SO, et al. Expression of heat shock proteins (HSP27, HSP60, HSP70, HSP90, GRP78, GRP94) in hepatitis B virus-related hepatocellular carcinomas and dysplastic nodules. World journal of gastroenterology: WJG. 2005;11(14):2072-2079.

33. Lebret T, et al. Heat shock proteins HSP27, HSP60, HSP70, and HSP90. Cancer. 2003;98(5):970-977.

34. Yano M, et al. Expression and roles of heat shock proteins in human breast cancer. Cancer Science. 1996;87(9):908-915.

35. Song $\mathrm{CH}$, et al. Potential prognostic value of heat-shock protein 90 in the presence of phosphatidylinositol-3-kinase overexpression or loss of PTEN, in invasive breast cancers. Breast Cancer Research. 2010;12(2):R20.

36. Huang B, et al. Differential expression of estrogen receptor $\alpha, \beta 1$, and $\beta 2$ in lobular and ductal breast can- 
cer. Proceedings of the National Academy of Sciences. 2014;111(5):1933-1938.

37. Barrios C, et al. What is the role of chemotherapy in estrogen receptor-positive, advanced breast cancer? Annals of oncology. 2009;20(7):1157-1162.

38. Taherian A, Mazoochi T. Different Expression of Extracellular Signal-Regulated Kinases (ERK) 1/2 and Phospho-Erk Proteins in MBA-MB-231 and MCF-7 Cells after Chemotherapy with Doxorubicin or Docetaxel. Iran J Basic Med Sci. 2012;15(1):669-77.

39. Taherian A, Krone PH, Ovsenek N. A comparison of Hsp90 $\alpha$ and Hsp90 $\beta$ interactions with cochaperones and substrates. Biochemistry and Cell Biology. 2008;86(1):3745.

40. Didelot $\mathrm{C}$, et al. Interaction of heat-shock protein $90 \beta$ isoform (HSP90 $\beta$ ) with cellular inhibitor of apoptosis 1 (c-IAP1) is required for cell differentiation. Cell Death Differ. 2008;15(5):859-66.

41. Dong H, et al. Breast cancer MDA-MB-231 cells use secreted heat shock protein-90alpha (Hsp90a) to survive a hostile hypoxic environment. Scientific reports. 2016;6.

42. Romaniuk A, Lyndin M. Immune microenvironment as a factor of breast cancer progression. Diagnostic pathology. 2015;10(1):79. 